\section{A PHASE IB STUDY OF THE SAFETY AND TOLERABILITY OF PIXATIMOD PLUS NIVOLUMAB IN SUBJECTS WITH ADVANCED SOLID TUMORS WITH AN EXPANSION COHORT IN METASTATIC PANCREATIC ADENOCARCINOMA (MPDAC)}

${ }^{1}$ Charlotte Lemech* ${ }^{*}{ }^{2}$ Keith Dredge, ${ }^{2}$ Darryn Bampton, ${ }^{2}$ Edward Hammond, ${ }^{3}$ Amanda Stanley, ${ }^{3}$ Lucie Leveque-ElMouttie, ${ }^{3}$ Grace Chojnowski, ${ }^{4}$ Andrew Haydon, ${ }^{5}$ Nick Pavlakis, ${ }^{6}$ Matthew Burge, ${ }^{7}$ Michael Brown, ${ }^{8}$ David Goldstein. ${ }^{1}$ Scientia Clinical Research Pty Ltd, Randwick, Australia; ${ }^{2}$ Zucero Therapeutics Ltd, Brisbane, Australia; ${ }^{3}$ QIMR Berghofer, Brisbane, Australia; ${ }^{4}$ The Alfred Hospital, Melbourne, Australia; ${ }^{5}$ Royal North Shore Hospital, Sydney, Australia; ${ }^{6}$ Royal Brisbane and Women's Hospital, Brisbane, Australia; ${ }^{7}$ Royal Adelaide Hospital, Adelaide, Australia; ${ }^{8}$ Prince of Wales Hospital, Sydney, Australia

Background Pixatimod is a novel immunomodulatory agent which stimulates dendritic cells (DC) via Toll-Like Receptor (TLR9) pathway to activate natural killer (NK) cells. ${ }^{1}$ In combination with PD1 inhibitors, it also enhances $\mathrm{T}$ cell infiltration in vivo. ${ }^{2}$ We report on safety, pharmacokinetics (PK) and pharmacodynamics (PD), and antitumor activity of pixatimod plus nivolumab in advanced cancer patients (stage 1) and in an expansion cohort of mPDAC (stage 2).

Methods In the dose escalation stage $(3+3$ design), eligible patients $(E C O G \leq 1)$ with advanced solid malignancies who failed standard therapies received pixatimod once weekly as a 1-hour i.v. infusion plus nivolumab $(240 \mathrm{mg}$, every other week) until disease progression or discontinuation due to intolerability. The primary objective was determination of the maximum tolerated dose (MTD). Secondary objectives evaluated safety, antitumor activity per RECIST v1.1, PK of pixatimod, and PD (PBMC, plasma cytokines and chemokines). Stage 2 comprised mPDAC subjects who had received no more than one prior line of chemotherapy in the metastatic setting.

Results The dose-escalation stage recruited 16 subjects across two cohorts ( $25 \& 50 \mathrm{mg}$ pixatimod). Two dose limiting toxicities (DLTs) in $50 \mathrm{mg}$ cohort were pulmonary edema and multi-organ failure. Of note, the subject with multi-organ failure had substantially higher CA19.9, Pan-immune-Inflammatory Value (PIV $=$ Neutrophils $\mathrm{x}$ Platelets $\mathrm{x}$ Monocytes/ Lymphocytes) and interleukins (IL) IL-1 $\alpha$ and IL-23 at baseline compared with the cohort. One DLT occurred in the 25 mg cohort, pneumonitis, which was identified as the MTD. A further $14 \mathrm{mPDAC}$ subjects were recruited to the expansion stage $(25 \mathrm{mg})$. Seven SAEs were reported to be possibly or likely related to the combination. No objective responses were reported in the mPDAC stage, the best response was SD ( $\mathrm{n}=$ 3). In another submitted abstract by Lemech et al, we report two subjects in the dose escalation stage with MSS mCRC were confirmed PR, and data from the amended study to include an MSS mCRC expansion cohort will also be presented. Time versus concentration data for pixatimod in advanced cancer patients was similar to that previously reported in monotherapy setting. In mPDAC subjects, there was minimal immune activation as evidenced by a lack of change in effector memory $\mathrm{T}$ cells or NK cells in PBMC, plasma cytokines and chemokines.

Conclusions Pixatimod is well tolerated at $25 \mathrm{mg}$ in combination with nivolumab but did not provide clear clinical benefit or evidence of immune activation in the mPDAC cohort.

Acknowledgements Research funding was provided by Zucero Therapeutics Ltd and Bristol Myers Squibb (BMS), Australia.

Trial Registration Clinical trial information: ACTRN12617001573347.

\section{REFERENCES}

1.. Brennan TV, Lin L, Brandstadter JD, Rendell VR, Dredge K, Huang $X$, et al. Heparan sulfate mimetic PG545-mediated antilymphoma effects require TLR9-dependent NK cell activation. J Clin Invest 2016;126(1):207-19.

2.. Hammond $E$, Haynes NM, Cullinane $C$, Brennan TV, Bampton D, Handley $P$, et al. Immunomodulatory activities of pixatimod: emerging nonclinical and clinical data, and its potential utility in combination with PD-1 inhibitors. J Immunother Cancer 2018;6(1):54

Ethics Approval The clinical trial entitled "An open-label, multi-centre Phase Ib study of the safety and tolerability of IV infused PG545 in combination with nivolumab in patients with advanced solid tumours with an expansion cohort in patients with metastatic pancreatic cancer. Protocol ZU545102" obtained ethics approval from the Royal Adelaide Hospital (HREC Reference number, HREC/17/RAH/195 and the CALHN Reference number, R20170515) and Bellberry Limited (Application No: 2018-08-695). All participants in the study gave informed consent before taking part in ZU545102.

http://dx.doi.org/10.1136/jitc-2021-SITC2021.340 